\title{
EVALUATION OF THE EFFECT OF HYALURONIC ACID MIXED WITH BIPHASIC CALCIUM PHOSPHATE ON BONE HEALING AROUND DENTAL IMPLANTS (EXPERIMENTAL STUDY)

\author{
Mostafa M. Shamma ${ }^{l} B D S$, Sherif S. Ayad ${ }^{2} P h D$, Riham M. El-dibany ${ }^{2} P h D$,
} Dina A. Nagui ${ }^{3} P h D$
}

\begin{abstract} osteopromoting materials that can be added to the BCP to enhance its osteoinductive properties. histological examination using Haematoxylin and eosin stain and Trichrome stain. new bone formation. The newly formed bone was more evident in association with group (A). osseous defects.

- B. D. S. Faculty of Dentistry, Alexandria University.

2- Professor of Oral \& Maxillofacial Surgery, Faculty of Dentistry, Alexandria University.

3- Lecturer of Oral Biology, Faculty of Dentistry, Alexandria University.
\end{abstract}

INTRODUCTION: Biphasic calcium phosphate (BCP) is very widely used as a grafting material around dental implants. The properties of such material can be enhanced by adding interpositional graft materials to enhance osteoinduction. Hyaluronic acid (HyA) is an example of

OBJECTIVES: Histological evaluation of using HyA with BCP on bone healing around dental implants.

MATERIALS AND METHODS: This study was a split mouth design. It was conducted on 9 mongrel dogs. The dogs were allocated into two groups: Group A (Study Group): The right side of the mandible received dental implants with biphasic calcium phosphate bone graft mixed with hyaluronic acid following extraction of the mandibular third premolar. Group B (Control Group): The left side of the mandible received dental implants with biphasic calcium phosphate bone graft only following extraction of the mandibular third premolar. Dogs were sacrificed at 2, 4 and 6 weeks postoperatively. Segments containing the implant and bone graft were retrieved with adjacent bone to be prepared for

RESULTS: All animals survived well, and remained active and alert all over the course of the experiment. Both groups were characterized by

CONCLUSIONS: HyA accelerates the onset of new bone formation when combined with BCP for bone augmentation in the treatment of

KEY WORDS: Dental implants, biphasic calcium phosphate, mandible, hyaluronic acid, osseointegration.

\section{INTRODUCTION}

Endosseous implants have provided a new dimension for replacing missing teeth, giving predictable long-term results in large patient population (1). Changes in implant shapes, sizes, materials, and coatings along with technique innovations, such as guided tissue regeneration and immediate loading concepts, have expanded the indications for dental implant therapy (2).

Since the first report of the immediate placement of a dental implant into a fresh extraction socket, there has been increasing interest in this technique for implant treatment (3). Numerous studies have reported that long-term (3-7 years) survival rates of dental implants immediately placed in a tooth extraction socket were similar to delayed implantation in healed extraction sites (4).

Graft materials and barrier membranes have been used extensively when placing immediate implants (5). Varieties of materials are used to fill this space between the implant and the surrounding socket walls. The fact that autogenous bone availability is limited led to development of alloplastic bone grafts. Biphasic calcium phosphate (BCP) is composed of different concentrations of the stable phase, hydroxyapatite (HA), and the more soluble phase, Betatricalcium phosphate $(\beta-\mathrm{TCP})$. It has presented significant advantages over other calcium phosphate ceramics due to their controlled bioactivity and balance between resorption/solubilization, which guarantees the stability of the biomaterial while promoting bone ingrowth (6).

Hyaluronic acid (HyA) is one of the essential components of extracellular matrix, which plays a predominant role in tissue morphogenesis, cell migration, differentiation, and adhesion. It has been recently reported that HyA increases osteoblastic bone formation in vitro through increased mesenchymal cell differentiation and migration (7).

Therefore, this study was conducted to evaluate the effect of hyaluronic acid mixed with biphasic calcium phosphate on bone healing and osseointegration around dental implants in dogs.

\section{MATERIALS AND METHODS \\ Research deign}

This study was approved from the ethical committee of the Faculty of Dentistry, Alexandria University. The study was a split mouth design.

This study was conducted on 9 mongrel dogs, 6 to 12 months of age and $10-15 \mathrm{Kg}$ in weight. All dogs were healthy as documented by a veterinarian report. The surgical procedure took place in the Physiology Department, Faculty of Medicine, Alexandria University. The dogs were allocated into two groups:

- Group A (Study Group): The right side of the mandible received dental implants with biphasic calcium phosphate bone graft mixed with hyaluronic acid following extraction of the mandibular third premolar.

- Group B (Control Group): The left side of the mandible received dental implants with biphasic calcium phosphate bone graft only following extraction of the mandibular third premolar. 


\section{MATERIALS}

\section{Biphasic calcium phosphate}

DMBone $^{\mathrm{TM}}$ (Meta Biomed,korea) is a bioactive bone graft material. It consists of two-phase ceramics: silicon contained coralline $60 \%$ hydroxyapatite and $40 \%$ betatricalcium phosphate. It is supplied in a vial containing $0.1 \mathrm{~mm}$ to $4.0 \mathrm{~mm}$ diameter granules.

\section{Hyaluronic acid}

Hyadent $^{\mathrm{TM}}$ (BioScience $\mathrm{GmbH}$-Germany) is a sterile, transparent and highly viscous gel obtained by crosslinking of special hyaluronic acid of non-animal origin. Two to three weeks after the application, the gel is completely reabsorbed. It is supplied in a package containing one syringe. The volume of the syringe is $1.0 \mathrm{ml}$ of hyaluronic acid in hydrogel form. (Fig.1)

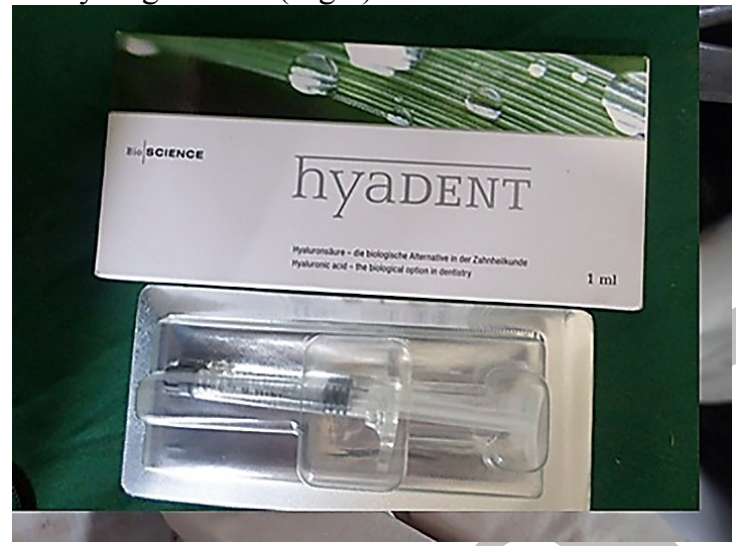

Figure (1): A photograph showing Hyadent

\section{METHODS}

\section{Presurgical phase}

All dogs were kept under the same environmental condition. The dogs received the same balanced diet through the period of the study (milk, meat, broth). All animals received a dose of antibiotic intramuscularly: ampicillin $25 \mathrm{mg} / \mathrm{kg}$ just before the operation. (Flumox, Eipico pharmaceutical Co. Cairo Egypt.)

\section{Surgical phase}

Each animal was generally anesthetized via intravenous injection of thiopentone sodium (Pentothal, Glazer exports Co. Dinshaw Waccha India.), the dose of which was calculated on the basis of $30 \mathrm{mg} / \mathrm{kg}$ body weight. The site of the operation was swabbed with $2 \%$ povidone-iodine (Povidone, Panax Pharma, PharmaCare, Egypt.) as an antiseptic. The mandibular third premolar was extracted so that implants can be placed in the socket immediately (Fig.2). Extraction sockets were prepared to receive the implants using sequential drills according to the manufacturer's instructions then the immediate implants were inserted followed by the bone graft as follows:

The study group (group A): the right side defect around the implant was filled with biphasic calcium phosphate (DM bone $^{\mathrm{TM}}$ ) mixed with hyaluronic acid (Hyadent ${ }^{\mathrm{TM}}$ ). (Fig.3)

The control group (group B): the left side was defect around the implant was filled with only biphasic calcium phosphate (DM bone ${ }^{\mathrm{TM}}$ ).

After the dental implants were placed, the buccal and lingual mucosa were approximated around the dental implant using 3-0 vicryl suture.

\section{Postsurgical phase}

All animals received the same course of antibiotics of ampicillin (Flumox, Eipico pharmaceutical Co.,Cairo Egypt.) $25 \mathrm{mg} / \mathrm{kg}$ body weight every eight hours for 5 days post operatively. Diclofenac potassium (Cataflam Novartis pharma, Cairo, Egypt) 25mg/day subcutaneous injection every eight hours was given as anti-inflammatory and analgesic for 3 days' post operatively.

Dogs were maintained on soft diet consisting of bread, milk and broth until the date of dog sacrification.

\section{Animal sacrification}

Three dogs were sacrificed at intervals of 2, 4 and 6 weeks postoperatively by giving them an overdose of thiopentone sodium intravenously. Segments containing the implant and bone graft were retrieved with the adjacent bone to be prepared for staining and histological examination.

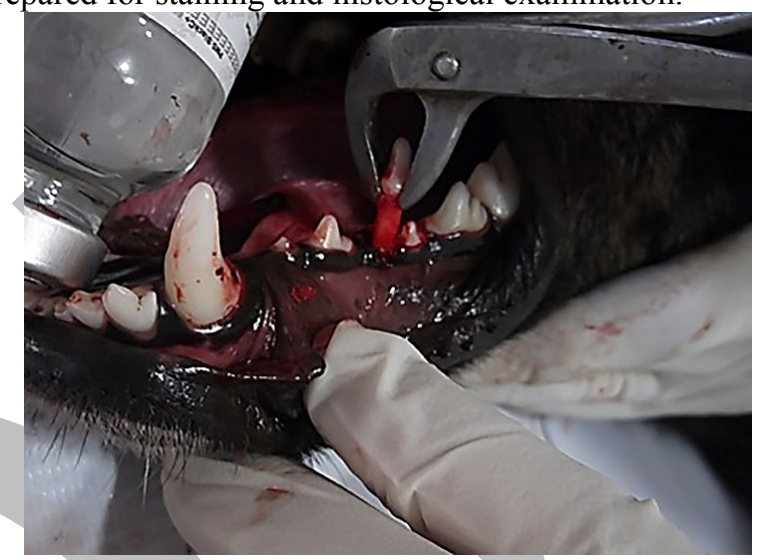

Figure (2): A photograph showing extraction of the lower third premolar.

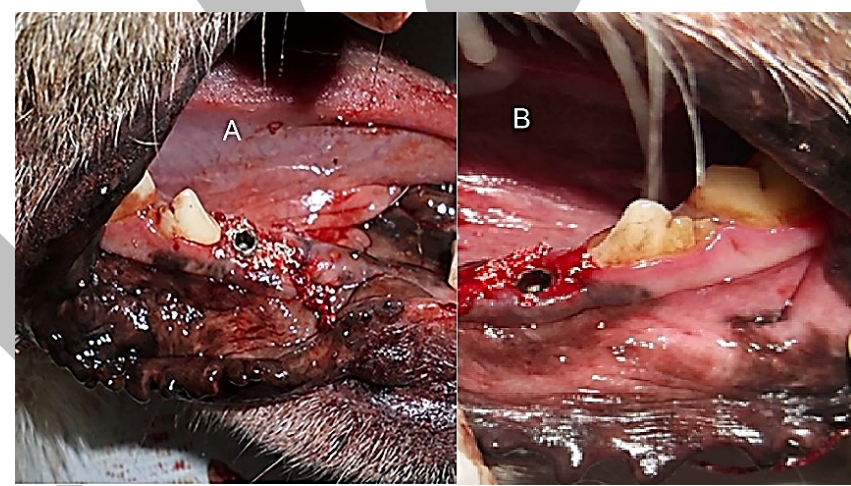

Figure (3): A: A photograph showing group $\mathrm{A}$ in which the defect around the implant was filled with DM bone graft mixed with Hyadent

B: A photograph showing group $B$ in which the defect around the implant was filled with DM bone graft only.

\section{RESULTS}

\section{Clinical results}

All animals survived very well and remained active and alert all over the course of the experiment. During the first week, the animals did not exhibit any clinical signs of infection and this period went without complications. Four weeks postoperatively, there were no sign of graft rejection in the bony defects created around the implant site, also no bony depressions were noted. Six weeks postoperatively, the continuity of the bone at the surgical site was the same at both sides.

\section{Histological Results}

Two weeks following the experimental procedures:

Group A: Areas of newly formed immature bone trabeculae of moderate thickness were interconnected to the native bone at the implant site. Numerous osteoblasts were seen uniformly arranged along the newly formed bone. (Fig.4A) Group B: The overall view of the histological 
observations here was generally similar to those observed in group A with some differences that included less amount of blood supply and less amount of the reactive newly formed bone. (Fig.4B)

Results obtained four weeks following the experimental procedures:

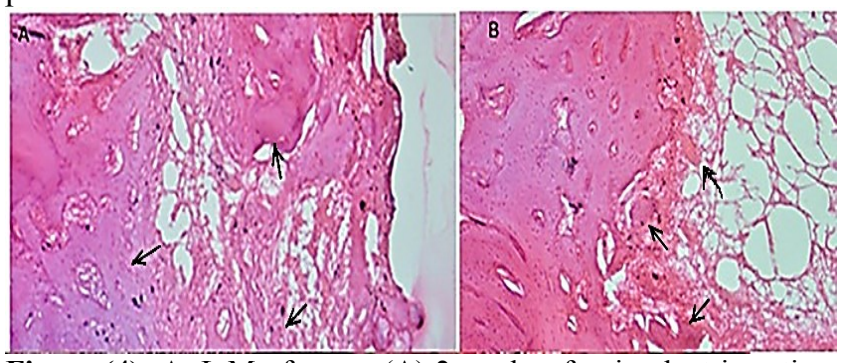

Figure (4): A: L.M of group (A) 2 weeks after implant insertion showing: dense granulation tissue formation, immature bone trabeculae appear within the dense collagen strands formed at the site of pre-existing implant (arrows). (H\&E stain, x100)

B: L.M of group (B) 2 weeks after implant insertion showing: granulation tissue formation with deposition of organic matrix of bone, small bony spicules are formed at the site of pre-existing implant (arrows). (H\&E stain, x100)

Group A: The healing defect consisted of newly formed mass of trabecular bone forming a continuous ring of dense trabeculae enclosing separate islands of connective tissue. The newly well-formed bone contained small to medium sized marrow spaces, high density of osteocytes and were bordered by osteoblasts (Fig.5A). Group B: There were increased amounts of collagen fibers seen between the newly formed bone trabeculae. In some specimens, the trabeculae appeared to be formed as isolated zones incompletely fused at this stage. (Fig.5B)

Results obtained six weeks following the experimental procedures:

Group A: The healing defect appeared completely filled by mature well-formed bone. The newly formed bone consisted of trabeculae extending towards the core of the defect with obvious complete osseointegration with the native bone. The new bone trabeculae were large and showed narrow marrow spaces. There was a notable difference in the number of osteocytes between the new bone and the native bone. (Fig.6A) Group B: The healing defect consisted of bone trabeculae of variable thickness extending into the defect center. The newly formed bone trabeculae contained medium-sized bone marrow spaces with remaining areas of granulation tissue. The newly formed bone trabeculae showed predominance of immature bone in relation to mature bone. (Fig.6B)

\section{DISCUSSION}

The combination of HyA with autogenous bone or bone substitute materials is opening the door to novel therapeutic alternatives and improving preexisting ones. Studies have demonstrated that HyA used to promote healing in both soft tissue and bone regeneration (8). In this study, HyA was mixed with $\mathrm{BCP}$ bone graft to increase bone healing around immediate implants placed in fresh extraction sockets with bony defects.

The cross-linked Hyaluronic Acid in the form of viscous gel allowed the HyA to bind to the granules of the bone graft easily and it was not resorbed before twelve to fourteen days (9).
It has been reported that HyA regenerative function is strongly affected by its physical properties. According to $\mathrm{N}$. Zhao et al. (10), the reticulated HyA can demonstrate a better regenerative function compared to linear HyA when it is mixed with $\beta$-TCP granules.

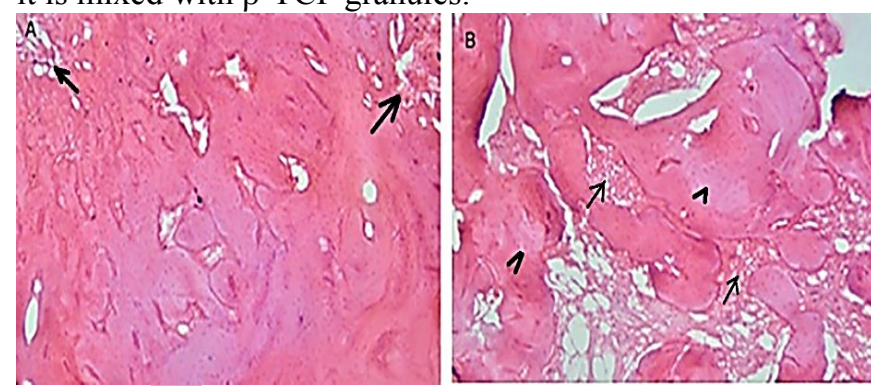

Figure (5): A: L.M of group (A) 4 weeks after implant insertion showing: well formed bone trabeculae containing small to medium-sized bone marrow spaces. Not the minimal amount of granulation tissue remaining at the borders of the new bone (arrows). (H\&E stain, x100)

B: L.M of group (B) 4 weeks after implant insertion showing: small amounts of collagen fibers seen between the new bone trabeculae (arrows). Note the numerous osteocytes densely arranged within the newly-formed bone (arrowheads). (H\&E stain, $\mathrm{x} 100$ )

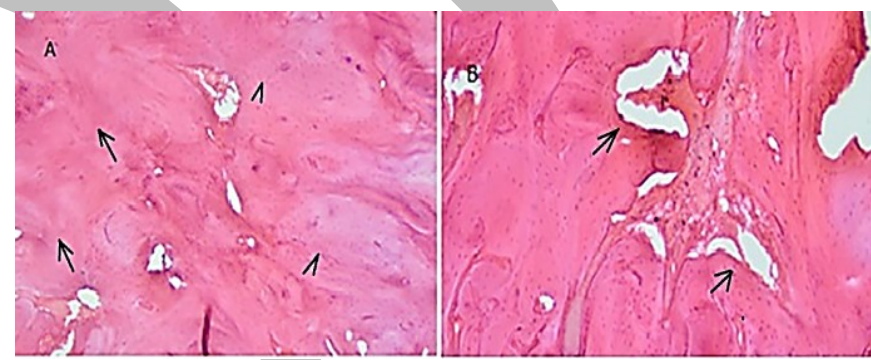

Figure (6): A: L.M of group (A) 6 weeks after implant insertion showing: Large newly- formed bone trabeculae with small-sized marrow spaces. Not the difference in the number of osteocytes between the old bone (arrows), and the new bone (arrowhead) (H\&E x100).

B: L.M of group (B) 6 weeks after implant insertion showing: well-formed bone with large marrow spaces containig dense granulation tissue (arrows)(H\&E x100).

The effectiveness of HyA depends not only on its features but also on the properties of co-administered grafting material. In this study, we used BCP as a bone graft to fill the defect around the immediate implant. Alloplastic grafting products such as (BCP) are synthetic in origin, biocompatible and osteoconductive (11). The release of controlled levels of calcium ions over time favors the formation of an appatite layer, which is necessary for the bioactivity displayed in BCP ceramics (12). This bioactivity can be responsible for the ceramic's osteoconductivity.

In accordance with our purpose of using BCP as a bone graft to fill the bony defect, Manjubala et al. in 2005 (13) studied the bone in-growth induced by BCP in femoral defect of dogs. Their results showed that the process of ossification started after 4 weeks of grafting, and the defect was completely filled with new woven bone after 12 weeks.

The results of this study showed that the newly wellformed bone contained small to medium sized marrow spaces. The remaining areas of granulation tissue appeared invaded by large engorged blood vessels. The blood supply and the newly formed bone, both were greater in group A than in group B. 
HyA likely increases cell migration, proliferation, and differentiation at the operative site, and enhance extracellular matrix organization. Furthermore, it stimulates newly formed capillaries surrounding subcutaneous implants (14).

In accordance to these findings, King et al. (15) have tested the influence of HyA on wound healing in rat cheek pouches. They have shown that exogenous HyA improved microcirculatory perfusion at the site of tissue repair in the wound area, and accelerated wound closure.

In the third observational period, the study group was characterized by the presence of mature well-formed bone. The newly formed bone consisted of trabeculae extending towards the core of the defect with obvious complete osseointegration with the native bone. The new bone trabeculae were large and showed narrow marrow spaces.

On the other hand, the control side showed bone trabeculae of variable thickness. The newly formed bone trabeculae contained medium-sized bone marrow spaces with remaining areas of granulation tissue. The newly formed bone trabeculae showed predominance of immature bone in relation to mature bone.

When in close contact with bone, HyA participates in bone morphogenesis and the early osteogenic events (16) modulating the effects of several cytokines and growth factors. HyA also induces bone formation similar to osteogenic substrates such as calcitonin and bone morphogenic protein. In addition, it binds proteins crucial for wound healing such as fibrinogen, fibrin, fibronectin, and collagen (17).

Similarly, in 2016, Kim et al. (18) applied HyA to fresh extraction sockets with previous pathology. They found out that HyA, because of its osteoinductive, bacteriostatic, and anti-inflammatory properties, may improve bone formation and accelerate wound healing in infected sockets.

On the other hand, Eric Aguado et al. in 2013 (19) investigated the use of hyaluronic acid (HyA) as an aqueous binder of the BCP bone graft granules. They made holes in the femoral condyle of each rabbit and filled some of them with BCP mixed with HyA while the other holes were filled only with BCP. Rabbits were allowed to heal for one month, sacrificed and femurs were harvested and analyzed. On contrary to our results, they found out that the amount of formed bone was not significantly higher than with BCP granules alone. HyA was rapidly eluted from the grafted zone but allowed deposition of more granules.

The positive results obtained in association with group A in all of the experimental periods suggest a superior effect of HyA and rapid start of bone formation when used in association with BCP as a bone graft than if used alone.

\section{CONCLUSIONS}

The combined use of HA and BCP for bone augmentation in the treatment of osseous defects is a potential treatment alternative for faster healing than using these biomaterials alone.

\section{CONFLICT OF INTEREST}

The authors declare that they have no conflict of interest.

\section{REFRENCES}

1. Bain C, Moy P. The association between the failure of dental implants and cigarette smoking. Int J Oral Maxillofac surg. 1993; 8: 609-15.
2. Puleo D, Thomas M. Implant surfaces. Dent Clin North Am. 2006; 50: 323-38.

3. David A, Mark V. Implant Surfaces. Dent Clin N Am. 2006; 50: 323-38.

4. Cooper L.F, Rahman A, Moriarty J, Chaffee N, Sacco D. Immediate mandibular rehabilitation with endosseous implants: Simultaneous extraction, implant placement and loading. Int J Oral Maxillofac surg. 2002; 17(4), 517-25.

5. Dahlin C, Sennerby L, Lekholm U, Linde A, Nyman S. Generation of new bone around titanium implants using a membrane technique: an experimental study in rabbits. Int J Oral Maxillofac Implants. 1989; 4: 19-25.

6. Tamini F, Torres J, Tresguerres I, Clemente C, Cabarcos E. Bone augmentation in rabbit calavaria: comparative study between Bio-Oss and a novel B-TCP/DCPD granulate J Clin Periodontol. 2006; 33: 922-8.

7. Fraser RE, Laurent TC, Laurent BG. Hyaluronan: Its Nature, Distribution, Functions and Turnover. J Intern Med 1997; 242: 27-33.

8. Stecco C, Stern R, Porzionato A, Macchi V, Masiero S, Stecco A, De Caro R. Hyaluronan within fascia in the etiology of myofascial pain. Surg Radiol Anat. 2011; 33(10): 891-6.

9. Tan H, Li H, Rubin J, Marra K. Controlled gelation and degradation rates of injectable hyaluronic acid based hydrogels through a double crosslinking strategy. J regen Med. 2011; 5(10): 790-7.

10. Zhao N, Wang X, Qin L. Effect of molecular weight and concentration of hyaluronan on cell proliferation and osteogenic differentiation in vitro. Biochem phys Res. 2015; 465(3): 569-74.

11. Bolukbsi N, Yeniyol S, Soluk M, Altunatmaz K. The use of platelet rich fibrin in combination with biphasic calcium phosphate in the treatment of bony defects: a histologic and histomorphometric study. Curr Therap Res. 2013; 75: 1521.

12. Daculsi G, LeGeros R. Encyclopedia of biomaterials and biomedical engineering, 2nd ed. New York: Informa Healthcare Inc. 2006; 359-66.

13. Manjubala I, Sastry T, Kumar R. Bone ingrowth induced by biphasic calcium phosphate ceramic in femoral defect of dogs. J Biomater Apppl. 2005; 19: 341-60.

14. West DC, Hampson IN, Arnold F, Kumar S. Angiogenesis induced by degradation products of hyaluronic acid. Science 1985; 228: 1324-6.

15. King S, Hickerson W, Proctor K, Newsome A. Benefical Actions of Exogenous Hyaluronic Acid on Wound Healing. Surgery. 1991; 109(1): 76-84.

16. Aslan M, Simsek G, Dayi E. The effect of hyaluronic acid supplemented bone graft in bone healing: experimental study in rabbits. J Biomater Appl 2006; 20: 209-20.

17. Stern M, Schmidt B, Dodson TB, Stern R, Kaban LB. Fetal cleft lip repair in rabbits: histology and role of hyaluronic acid. J Oral Maxillofac Surg 1992; 50: 263-9.

18. Kim JJ, Song HY, Ben Amara H, Kyung-Rim K, Koo KT. Hyaluronic Acid Improves Bone Formation in Extraction Sockets with Chronic Pathology: A Pilot Study in Dogs. J Periodontol. 2016; 87(7): 790-5.

19. Aguado E, Florence P, Christine G, Eric G, Daniel C. $\beta$ TCP granules mixed with reticulated hyaluronic acid induces an increase in bone apposition. Biomed Mater. 2013; 9(1): 015001. 